\title{
Investigation of Factors Involved in the Uptake Velocity of Fluorescein Diacetate and Intracellular Fluorescence Polarization Value. I. Physiological Aspects in Lymphoblastoid Cells
}

\author{
Hiroyuki Tsuda ${ }^{1)}$ and Hiroshi Maeda ${ }^{2) *}$ \\ The Second Department of Medicine ${ }^{1)}$ and Department of Microbiology ${ }^{2}$, \\ Kumamoto University Medical School, Kumamoto 860, Japan
}

\begin{abstract}
Measurement of cellular fluidity by the fluorescence polarization method with a fluorogenic dye, fluorescein diacetate (FDA), is used widely. But values obtained with this method may be affected greatly by unknown factors. To determine the various factors that affect fluorescence polarization (FP-) measurements and intrinsic cellular fluidity, we investigated the uptake of FDA into cells, cellular esterases, fluorescein-binding protein, microtubules and osmolarity. Our results show that some reagents inhibit FDA-uptake without affecting esterases and that several types of esterases are involved in FDA-hydrolysis.

The dye-binding experiment indicated that dye-binding protein is absent from the cells. In addition, to determine whether there is involvement of microtubules in cellular fluidity, we treated cells with $\mathrm{Ca}^{2+}$ antagonists, calmodulin inhibitors and low temperature, and verified that there is little evidence for that hypothesis.

In other experiments, cells were brought into a hypertonic condition, which resulted in osmolysis and higher intracellular concentrations, primarily of biopolymers, and higher viscosity. The effect of this viscosity on the FP value was investigated. Results showed a parallel relationship between osmolarity and therefore, intracellular viscosity and FP value. In conclusion, the concentrations of intracellular proteins and other biopolymers appear to be a major factor that determines cellular fluidity, but the role of microtubules which undergo assembly-disassembly appears to be insignificant.
\end{abstract}

Rotman and Papermaster reported previously that fluorescein diacetate (FDA) is incorporated into cells only when they are viable (17) and that this incorporation may depend on facilitated active transport. Based on this finding, FDA, which be-

Abbreviations used: FDA, fluorescein diacetate; FP, fluorescence polarization; AML, acute myelogenous leukemia; CLL, chronic lymphocytic leukemia; IFP, intracellular fluorescence polarization; PBS, $0.01 \mathrm{M}$ phosphate buffered saline; BSA, bovine serum albumin; NCS, neocarzinostatin; BGG, bovine gamma globulin; BD, blue dextran; ANS, 8-anilino-1-naphtalene sulfonate. NEM, N-ethylmaleimide; DFP, diisopropylfluorophosphate; FOY-305, N,N-dimethylamino $p$-( $p^{\prime}$-guanidinobenzoyloxy) benzylcarbonyloxy glycolate.

* Address correspondence and reprints requests to $\mathrm{H}$. Maeda. 
comes a highly efficient fluorescent emitter in the cells, has been used widely as an intracellular probe for fluorescence polarization (FP) measurements of viable cells. We have now extended and quantitated this observation and found that the rate of uptake of FDA into cells was much affected by various cytotoxic agents as well as by the pathological conditions of the cells; thus, the rate of uptake would reflect the degree of cytotoxicity. Similarly, we anticipated that the FP values also would be affected by exposing the cells to cytotoxic conditions. Both of these parameters may produce quantitative data on cytotoxicity (13).

The FP method is known to provide information on the immediate environment of intracellular fluorescein molecules. Although the FP value is believed to reflect intracellular fluidity, little is known about the physical determinants of the FP value in cells $(3,17)$. This value, however, has been used widely, as in quantitation of the lymphocyte-response to treatment of plant mitogens that revealed impaired immunological competences of tumor patients $(3,19,21)$ and in differentiation of the lineage of leukemic cells (23).

The FP method should provide useful information concerning the physiological state of cells and their various responses. But, to our knowledge there is no report as to the effect of changes in FDA-uptake on FP values for cells exposed to cytotoxic conditions. To clarify this point we studied various factors which might affect these measurements.

The interpretation of such measurements in living cells is very complex indeed and vulnerable to changes in various factors. Cercek and Cercek (3) speculated on the possibility that changes in the FP value might be reflecting forces of interaction between macromolecules such as proteins and small molecules including water molecules, ions, ATP, and cyclic-AMP. We observed that the FP value of cells changes significantly after an addition of FDA to the milieu $(21,23)$. The reason for this gradual rise and fall in the value of FP is not known although the intracellular concentration of the probe as well as its subcellular localization obviously changes with time, in addition to leakage from the cells. We know that the extracellular leak contributes no more than $15 \%$ of the fluorescence intensity measured within 20 min at or below $30^{\circ} \mathrm{C}$ in lymphoblastoid cells. In any case, the rate of uptake of FDA and its hydrolysis to fluorescein in cells, and therefore its intracellular concentration, also needs to be analyzed. Accordingly, by elaborating on the procedural, biochemical and cellular events described above, measurements would become more precise and would be more reproducible, which would lead to more general applications. Based on these results, this study has been extended to various anticancer agents as described in the accompanying article (13).

\section{MATERIALS AND METHODS}

Chemicals. FDA was obtained from Dojin Chemical Leboratories, Kumamoto, Japan. $\mathrm{Ca}^{2+}$ antagonists and the calmodulin inhibitors verapamil, diltiazem and T-233 $(7,16)$ were gifts from Prof. K. Nishi (Department of Pharmacology of Kumamoto University) and Dr. R. Maruyama (Mitsubishi Kasei Institute for Life Science, Tokyo). 8-Anilino-1-naphthalene sulfonic acid sodium salt (ANS) and calf thymus histone were from Tokyo Kasei Industry Co., Ltd., Tokyo. All other chemicals were from commercial sources.

Instrument. Fluorescence intensity and FP value measurements were carried out with a Model MAC-2 polarization spectrofluorometer (Japan Immunoresearch Co., Ltd., Taka- 
saki) after some modifications; automatic quantification for the fluorescence intensity and the print-out. This instrument is water-jacketed for a constant temperature at $30^{\circ} \mathrm{C} \pm 0.05$ and uses a rotating emission polarizer which allows detection of both parallel and perpendicular fluorescence components by a single photomultiplier tube as the cosine function of the rotational time of the polarizer. The FP value is defined as $\left(I_{A}-I_{B}\right) /\left(I_{A}+I_{B}\right)$, in which $I_{A}$ and $I_{B}$ are the fluorescence intensity in the parallel and perpendicular modes respectively, and the value is printed out automatically as the average of 100 readings per $50 \mathrm{sec}$. The instrument has three-cavity filters (Ditric Optics, Hudson, Mass., U.S.A.), $490 \mathrm{~nm}$ for excitation and $520 \mathrm{~nm}$ for emission, which meet the spectroscopic requirements of fluorescein fluorophore and which permit passage of near monochromatic light with little depolarization $(\mathrm{T}=50 \%$ at 490 and $520 \mathrm{~nm}$, respectively).

Cells. The lymphoblastoid cell lines used were Daudi, P3HR-1, Ramos and C-6. They were cultured in RPMI-1640 medium enriched with $10 \%$ fetal calf serum and used during exponential growth, most typically within two to three days after inoculation. Viability was more than $95 \%$. In the experinent with fluorescein-binding proteins, P3HR-1 and leukemic cells from the patients with acute myelogenous leukemia (AML) and chronic lymphocytic leukemia (CLL) were used as well. These cells are known to have very different FP values each other (23).

Measurement of intracellular fluorescence polarization (IFP) with fluorescein as a probe. Cells in culture medium were spun down and washed twice with $0.01 \mathrm{M}$ phosphate buffered $0.15 \mathrm{M} \mathrm{NaCl}$ (PBS, pH 6.9). The pelleted cells then were resuspended in PBS, or in PBS of varying osmolarity adjusted with $\mathrm{NaCl}$ in special cases, followed by adjustment of the cell density to $5 \times 10^{5} / \mathrm{ml}$. This gave an absorbance of no more than 0.03 in the $490-520 \mathrm{~nm}$ range (19). A 2-ml sample of the cell-suspension then was inserted and $50 \mu$ of the FDA stock solution, which had been prepared as a dispersion of $25 \mu \mathrm{M}$ in PBS, was added. The mixture was stirred quickly and spectroscopy was started.

When the effect of temperature on IFP was investigated, the cell suspension in PBS was incubated with FDA in a cuvette at $30^{\circ} \mathrm{C}$ for 3 min to facilitate rapid uptake of FDA into the cells. The suspension then was adjusted to the desired temperature by immersing it in a water bath after which the FP value was measured.

For the study of $\mathrm{Ca}^{2+}$ antagonists and calmodulin inhibitors, cells were treated with the respective drugs in the culture medium for $1 \mathrm{~h}$ after which they were removed, or in PBS for 5 min before the addition of FDA. In the latter case, Mersurements were carried out in the presence of the drugs in the cell suspension. In all other cases the cells were washed twice by pelletting them at $1,500 \mathrm{rpm}$ for $10 \mathrm{~min}$ in PBS that did not contain serum components nor the respective drugs. After this treatment they were resuspended in PBS.

For colchicine treatment, cells were first incubated with colchicine in the culture medium for $30 \mathrm{~min}$ at $37^{\circ} \mathrm{C}$ before measurement. For efficient and reproducible experiments, the times required for measurements, centrifugation and incubation were coordinated so that all the cells could be measured under an identical test condition.

Dye-binding experiment: Fluorescein binding with intracellular proteins. The noncovalent interaction of cellular proteins with fluorescein molecules (probe) was first investigated with gel filtration as reported previously $(8,10)$. Cell suspensions in PBS in test tubes were placed in an ice-chilled water bath, after which they were homogenized by sonication then centrifuged at $18,000 \times g$ for $10 \mathrm{~min}$. The supernatant was adjusted to give an optical density of 5.0 at $280 \mathrm{~nm}$, and diluted with the same volume of $8 \mu \mathrm{M}$ fluorescein solution in PBS. The solutions of bovine serum albumin (BSA), bovine gamma globulin (BGG), ovomucoid and calf thymus histone in PBS with $4 \mu \mathrm{M}$ fluorescein were prepared to give the same final optical density. 
These clear cell homogenates or protein solutions were applied to a column of Sephadex G-50 $(2.5 \times 68 \mathrm{~cm})$ which had been equilibrated beforehand with $4 \mu \mathrm{M}$ fluorescein solution in PBS. The column was used again similarly with $0.65 \mathrm{M} \mathrm{KCl}$ in PBS for the second experiment. The absorbances of the eluates $(2.80 \mathrm{ml} /$ tube $)$ from the Sephadex column were measured continuously at 280 and $490 \mathrm{~nm}$. When fluorescein bound to a protein, the bound fluorescein with increased absorption at $490 \mathrm{~nm}$, emerged with it as a discrete protein peak at $280 \mathrm{~nm}$.

We next examined the dye-binding property of cellular protein based on its FP value. A solution of cell sonicates prepared as above was diluted to give an absorbance of 2.0, 1.0 and 0.5 at $280 \mathrm{~nm}$ with PBS. BSA and histone were dissolved in PBS and adjusted to the corresponding concentrations based on the absorbance at $280 \mathrm{~nm}$. A $20-\mu 1$ sample of $0.5 \mu \mathrm{M}$ fluorescein solution in PBS was added to $1 \mathrm{ml}$ of each protein solution, then $0.5 \mathrm{ml}$ of this solution was transfered to a semi-microcuvette and the FP value was measured. In separate experiments, $500 \mu \mathrm{g}$ of ANS was added prior to the addition of fluorescein to the cuvette. ANS competes for the dye-binding site of fluorescein in BSA, which produces more unbound fluorescein and a lower FP value. The FP measurement was carried out at $30^{\circ} \mathrm{C}$ for more than $10 \mathrm{~min}$.

Effect of enzyme inhibitors on cellular esterases in a cell-free system. The enzyme inhibitors; N-ethylmaleimide (NEM), diisopropylfluorophosphate (DFP), antipain, leupeptin, $\gamma$-glucaro- $\delta$-lactum (25), $\mathrm{N}, \mathrm{N}$-dimethylamino $p$-( $p^{\prime}$-guanidinobenzoyloxy)-benzylcarbonyloxy glycolate (FOY-305) (22) and some cytotoxic agents were checked to see whether they affected the activity of cellular esterases for the hydrolysis of FDA. A 50- $\mu$ l sample of each drug in solution at varying concentrations was added to the cell-sonicates as described in the preceeding section, after which $5 \mu$ l of FDA stock solution was added. Measurement of the fluorescence intensity was resumed immediately and was continued for more than 20 min at $30^{\circ} \mathrm{C}$. The percent of inhibition of esterase activity was calculated as follows: $(\%$ of inhibition)- $\left(1-\mathrm{T}_{0} / \mathrm{T}_{\mathrm{i}}\right) \times 100$, in which $\mathrm{T}_{\mathrm{i}}$ and $\mathrm{T}_{0}$ are the respective times required to obtain 10 $\mathrm{pmol} / \mathrm{ml}$ of fluorescein with or without inhibitor.

\section{RESULTS}

Inhibition of cellular esterases. The apparent uptake velocity of FDA shown by the increase in fluorescence intensity consists of two rate limiting steps: (a) membrane transport and (b) hydrolysis of FDA. We now know that many drugs including the cytotoxic agents, NEM and DFP inhibit either step.

To analyze this inhibition, we investigated the effect of these drugs on cellular esterases (step b). Cell homogenates were treated with various drugs before the addition of FDA. Esterase activity was partially suppressed by NEM and DFP (Table 1). These inhibitors act additively when used simultaneously. Other inhibitors of microbial origin (Table 1) (25) and several anticancer agents were not effective at the concentrations tested, which suggests that the observed suppression of the fluorescence intensity in the intact cell system by various anticancer agents (13) may reflect the suppression of uptake velocity (step a) rather than hydrolysis.

Interaction of fluorescein and intracellular protein. When free fluorescein binds to any protein, the FP value becomes higher as a result of impeded rotational Brownian motion of fluorescein molecules $(11,12)$ : thus, the FP value may not always reflect intracellular fluidity. We thus examined the possibility of the formation of a dye (fluorescein)-protein complex. BSA, which represents the fluorescein-binding protein, 
TABLE 1. EFFECTS OF VARIOUS ESTERASE INHIBITORS AND ANTICANCER AGENTS ON CELLULAR ESTERASES

\begin{tabular}{|c|c|c|c|c|c|}
\hline & \multicolumn{5}{|c|}{ Drug concentration $(\mu \mathrm{g} / \mathrm{ml})$} \\
\hline & 10 & 25 & 50 & 100 & 200 \\
\hline & \multicolumn{5}{|c|}{ Esterase inhibition (\%) } \\
\hline \multicolumn{6}{|l|}{ (A) Enzyme inhibitors } \\
\hline NEM & 26.5 & - & 30.2 & 32.0 & 28.9 \\
\hline DFP & 7.9 & - & 18.1 & 40.9 & 53.0 \\
\hline $\mathrm{NEM}+\mathrm{DFP}$ & - & - & 53.7 & 63.1 & - \\
\hline Antipain & - & - & - & 9.6 & 9.6 \\
\hline Leupeptin & - & - & - & -4.2 & 11.3 \\
\hline$\beta$-Glucaro- $\delta$-lactum & - & - & - & -2.4 & -6.5 \\
\hline FOY-305 & - & -27.3 & - & - & -18.8 \\
\hline \multicolumn{6}{|l|}{ (B) Anticancer agents $\#$} \\
\hline Mitomycin C & 3.6 & - & - & - & - \\
\hline Neocarzinostatin & - & 2.4 & - & - & - \\
\hline Carboquone & 一 & - & - & 1.1 & - \\
\hline Methotrexate & 4.2 & - & - & - & - \\
\hline Cytosine arabinoside & -11.4 & - & - & - & - \\
\hline
\end{tabular}

\# The dosages correspond to the maximal concentrations used in the cytotoxicity assay (13).

formed a complex with the dye as shown in Fig. 1A; thus, the eluate showed a higher absorbance at both 490 and $280 \mathrm{~nm}$, whereas BGG and ovomucoid which represent the non-binding protein, showed no such effect (Fig. 1A).

The homogenates of P3HR-1, AML and CLL cells showed small increments in their absorbances which means there was the slight possibility of the presence of fluorescein-binding proteins within the cells, but the increments disappeared in $0.65 \mathrm{M}$ $\mathrm{KCl}$ solution (Fig. 1B). These small increments were, however, of about the same magnitude (data not shown) for two different cell origins, AML or CLL, regardless of the pronounced difference in the intracellular polarization values (IFP) (23).

To find the origin of the slight increment in absorbance at $490 \mathrm{~nm}$ we examined a basic protein, histone (Fig. 1C). The results showed that histone slightly induced the increased absorbance due to fluorescein, indicative of the interaction of fluorescein with histone molecules in PBS. This effect on P3HR-1, AML, CLL and histone was abolished by increasing the ionic strength to $0.65 \mathrm{M}$ with $\mathrm{KCl}$. Thus, the slight increments described above apparently are brought about by ionic interaction.

Another line of experiment based on the assumption that an increase in the IFP value meant the presence of dye-binding was made with cell homogenates. The effect of cellular protein on the polarization value of fluorescein at various protein concentration is shown in Fig. 2. When BSA was added to the solution, the rise in the FP value paralleled the dose of BSA, which signifies dye-binding with BSA. This relationship, however, was suppressed competitively by the addition of ANS. When cellulad proteins from myelogenous or lymphocytic leukemic cells or histone were examined no increases were found (Fig. 2) even at high concentrations of proteins. These results rule out the possibility of the presence of fluorescein-binding proteins which could affect the IFP value. 




Fig. 1. Fluorescein binding experiments on a column of Sephadex G-50. (A) Model experiments with bovine serum albumin (BSA), immunoglobulin (BGG) and ovomucoid, (B) cell homogenates of a lymphoblastoid cell line, P3HR-1 cells, and (C) calf-thymus histone. In each experiment the column first had been equilibrated with fluorescein to give an absorbance of 0.234 at $490 \mathrm{~nm}$ in PBS. In (A) the reading at $490 \mathrm{~nm}$ for BSA is shown by $(\bigcirc)$ and for ovomucoid by $(\triangle)$, and their readings at $280 \mathrm{~nm}$ are shown by $(\bullet)$ and $(\Delta)$. The values for BGG and ovomucoid were almost identical. BD (blue dextran) and NCS (neocarzinostatin) are molecular markers of $2 \times 10^{6}$ and $1 \times 10^{4}$ daltons, respectively, as shown by the arrows. In (B) and (C) the $490 \mathrm{~nm}$ readings are shown by $(\bigcirc)$ in PBS, and by $(\triangle)$ in $0.65 \mathrm{M} \mathrm{KCl}$, and the $280 \mathrm{~nm}$ readings by $(\bullet)$ in PBs.

Effect of treatments that affect microtubules on IFP. Microtubules are ubiquitous components of eukaryotic cells, intimately associated with many important cellular processes such as motility, development, and maintenance of cell-morphology $(5,20)$. Two experiments were carried out on the relationship of microtubules to IFP.

We first examined the effect of temperature on IFP because microtubules are in equilibrium with their subunits; low temperature $\left(<10^{\circ} \mathrm{C}\right)$ shifts the equilibrium toward disassembly to the monomeric form and high temperature $\left(>10^{\circ} \mathrm{C}\right)$ shifts it 


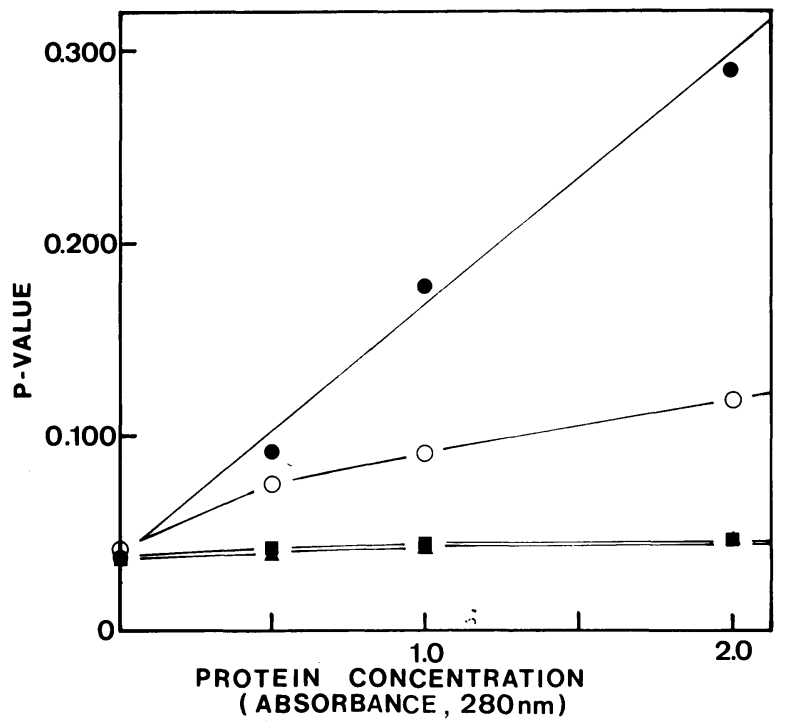

Fig. 2. Fluorescein binding experiments at different protein concentrations and polarization values. Protein concentrations were based on the absorbance at $280 \mathrm{~nm}$. $\bullet$, bovine serum albumin (BSA); $\bigcirc$, BSA treated first with aniline naphthalene sulfonate; $\mathbf{\square}$, cell homogenates of myelogenous or lymphocytic leukemic cells (values were almost identical); $\boldsymbol{\Delta}$, histone. The concentration of fluorescein was $0.01 \mu \mathrm{M}$ in PBS.

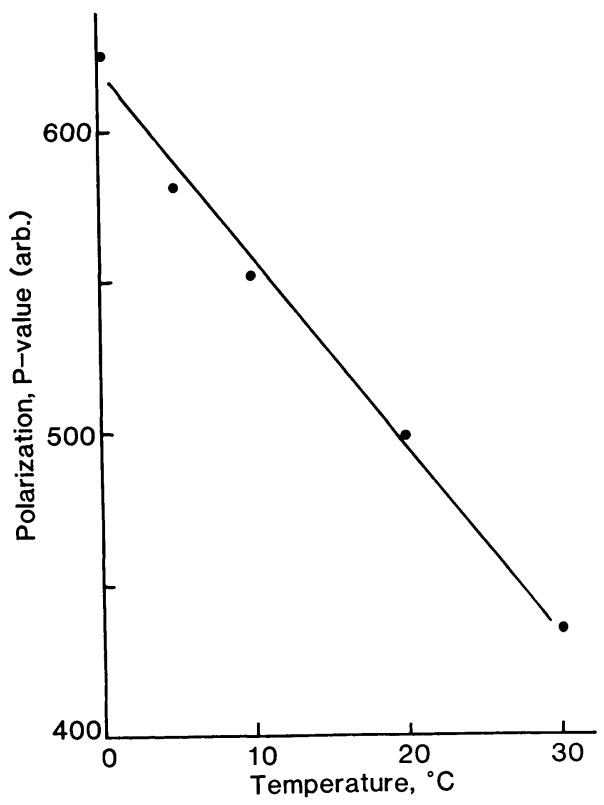

Fig. 3. Intracellular fluorescence polarization value with FDA and the effect of cellular temperature. Cells: C-6. All conditions are described in Methods. 


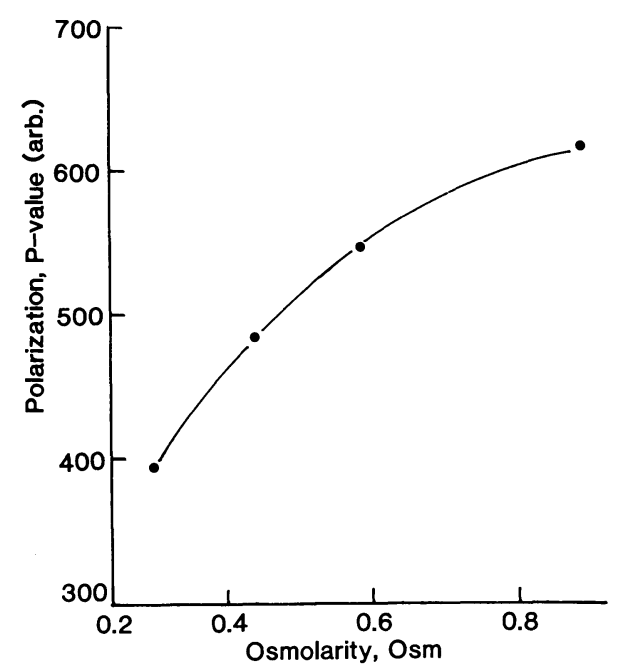

Fig. 4. Intracellular polarization value with FDA at various osmolarities in the assay medium. Cel[s: C-6.

toward assembly $(5,20)$. Our result showed, however, that the higher the temperature the lower the IFP value, namely a reverse linear relationship between temperature and the IFP value (Fig. 3). This result is similar to the model system of fluorescein in glycerol without microtubules. No inflection point was observed. Colchicine treatment (at $10^{-5} \mathrm{M}$, for $30 \mathrm{~min}$ ), which also is known to shift the equilibrium toward disassembly $(5,20)$, produced almost no change in IFP (data not shown).

A second experiment was made in view of $\mathrm{Ca}^{2+}$ and calmodulin using their antagonists or inhibitors since the calcium-calmodulin complex is known to prevent the assembly of microtubule protein in vitro (14). Our results indicated that the changes induced in the IFP value were negligible (less than $5 \%$ ) when the drugs verapamil $(500 \mu \mathrm{g} / \mathrm{ml})$, diltiazem $\left(10^{-4} \mathrm{M}\right)$, EGTA $\left(10^{-3} \mathrm{M}\right)$, streptomycin $(2 \mathrm{mg} / \mathrm{ml})(15)$ and TI-233 (100 $\mu \mathrm{M})(16)$ were used (data not shown).

Effect of osmolarity on IFP. The viscosity of a given solution is proportional to the concentration of responsible solute. In case of cytoplasmic viscosity it is likely to be influenced by protein, nucleic acid and other biopolymers. To obtain a high intracellular concentration of these macromolecules we exposed cells to high osmolarity which resulted in osmolysis producing a high intracellular concentration.

The IFP values of the fluorescein molecules in these cells that were measured at $37^{\circ} \mathrm{C}$ in PBS at different osmolarities are shown in Fig. 4. As osmolarity increased, the IFP value increased as well. The curve was very similar to that obtained for the FP values of fluorescein in glycerin and other proteins at varying corresponding concentrations (or osmolarities) (data not shown). Therefore, the increased IFP value under hypertonic conditions appears to reflect the increased macromolecular concentration within the cell.

\section{DISCUSSION}

The FP value is used to describe the physical state of organization of the cytoplasmic matrix, for which Cercek (3) has proposed the term "structuredness of the 
cytoplasmic matrix (SCM)'". This term is, however, vague and somewhat misleading because the method does not necessarily measure the state of the matrix-structure or the cytoskeleton directly. Nevertheless, the measurement of the IFP value is relatively easy and sensitive and it appears to be very useful in the analysis of intracellular fluidity and thus cell functions under various conditions. For instance, we now know, as described in the following article (13), that many cytotoxic agents including anticancer agents do affect the IFP value. We also know that the rate of increase in fluorescence intensity is affected greatly by the same treatment. But so far, the type of factors attributable to these changes have not been determined. The experiments reported here were done to determine the biochemical and cytological factors which influence these IFP measurements so that a better interpretation of the meaning of the IFP values can be made.

After FDA has been added to cells the first event is the uptake and increase in fluorescence intensity $(19,23)$. The increment in intensity is affected by various factors. As described above the process can be divided into two rate-limiting steps: the incorporation of FDA into the cells and its hydrolysis. The hydrolysis of FDA, which generates fluorescein (second step), is affected by few if any of the esterase inhibitors, and only to a limited extent by DFP which inactivates serine-type esterases and by NEM which inactivates sulfhydryl type esterases (Table 1).

These results suggest that there are at least three types of esterases involved in FDA hydrolysis, including the A- and B-esterases of Aldridge (1). Many other cytotoxic agents that strongly inhibited the increase in fluorescence intensity at the cellular level after an FDA addition did not inhibit esterase activity in the cell-homogenate (13). Therefore, the inhibition of fluorescence intensity brought about by these reagents in the cell system can be attributed to suppression of the transport system; the first step.

The possible presence of fluorescein-binding protein(s) that might affect the rotational Brownian motion of fluorescein molecules in the cells was investigated. Udkoff et al. deduced the presence of such binding from the relationship of the FP value to fluorescence intensity (24). We also confirmed it by a different method in which a Sephadex column was used (Fig. 1). When cellular protein or histone was applied to the Sephadex column, fluorescein was eluted in the protein peak (Fig. 1). This indicates that positively charged protein(s) absorbed negatively charged fluorescein, perhaps by ionic interactions. This supposition is further supported by the fact that under increased ionic strength $(0.65 \mathrm{M} \mathrm{KCl})$ fluorescein binding was reduced, probably by ionic competition. In addition, the FP-method produced direct substantiation in that FP values were influenced very little by an increased dose of cellular protein in the fluorescein solution. The opposite was observed for BSA, a well-known dye-binding protein (Fig. 2). Therefore, the binding of fluorescein to cellular protein, if present, may be loose enough to allow the free, Brownian rotational motion of fluorescein molecules as in the ionic interaction exemplified by histone (Fig. 1, 2).

The microtubule, because it is the probable candidate for the cytoplasmic structural organella which could affect IFP, was investigated. But, measurements of IFP values at various temperatures did not support this assumption. Low temperature treatment did not affect the linear relationship between temperature and the FP value (Fig. 3). This result is in contradiction with the assumption that low temperature facilitates the disassembly of tubulins which would result in a low IFP. Furthermore, vincristine, colchicine, and various $\mathrm{Ca}^{2+}$-antagonists or calmodulin inhibitors, all of which 
inhibit tubulin assembly $(14,20)$, produced no significant change in the IFP values. This is the evidence that there is no support for the idea that microtubules have a great influence on IFP and, hence, on intracellular viscosity.

The effect of intracellular viscosity on IFP value was investigated under different osmotic pressure and hence different cytoplasmic concentration of protein and other macromolecules. When the ionic strength of the surrounding milieu is higher (hypertonic) than that of the cell sap, water leaks out of the cell and a higher cytoplasmic concentration results. Since viscosity is determined by the interaction between solute molecules, this increased intracellular concentration produces a higher viscosity and, thus, a higher IFP value. The results shown in Fig. 4 are in good agreement with this explanation and with the results of Cercek and Cercek (4).

We have shown that the FDA introduced into cells is hydrolyzed by several kinds of intracellular esterases which are inhibitable by DFP and NEM. Many other chemicals, however, which did not affect the esterases, did inhibit an increase in fluorescence intensity. Thus, FDA-uptake, rather than esterase activity, is more vulnerable to many of these chemicals. IFP seems to be influenced neither by the microtubules nor by the fluorescein-binding proteins in cells, but the intracellular viscosity has a major influence on the IFP value.

Recently, Rotman et al. (18) reported that the decrease in the cytoplasmic viscosity of the platelet after activation by thrombin is due to the conversion of actin to insoluble or compartmentable F-actin. Actin also is known to play an important role in the sol-gel transformation of various cells or extracts (9). In a recent study (6) the content of tropomyocin in transformed cells was found to be decreased and this may have a similar effect on cellular fluidity. We have not yet tested these possibilities, but the contractile proteins probably do play an important role in determining cellular fluidity.

Acknowledgment. This work was supported by a Grant-in-Aid for Cancer Research 1982 from the Ministry of Education, Science and Culture of Japan.

\section{REFERENCES}

1. Bergman, F., R. Segal and S. Rimon. A new type of esterase in hog-kidney extract. Biochem. J. 67, 481-486, 1957

2. Brodrick, J.W., C.B. Galser, C. Largman, M.C. Geokas, M. Graceffo, M. Fassett and H. MAEDA. Interaction of chymotrypsinogen with $\alpha_{1}$-protease inhibitor. Biochemistry 19, 48654870, 1980

3. Cercek, L. and B. Cercek. Application of the phenomenon of changes in the structuredness of cytoplasmic matrix (SCM) in the diagnosis of malignant disorders: A review. Eur. J. Cancer 13, 903-915, 1977

4. Cercek, L. and B. Cercek. Effects of osmolarity, calcium and magnesium ions on the structuredness of cytoplasmic matrix. Rad. and Environm. Biophys. 13, 9-12, 1976

5. Dustin, P. Microtubules. Scientific American 243, 59-68, 1980

6. Hendricks, M. and H. Weintraub. Tropomyosin is decreased in transformed cells. Proc. Natl. Acad. Sci. U.S.A. 78, 5633-5637, 1981

7. Hidaka, H., T. Yamaki, M. Naka, T. Tanaka, H. Hayashi and R. Kobayashi. Calciumregulated modulator protein interacting agents inhibit smooth muscle calcium-stimulated protein kinase and ATPase. Mol. Pharmacol. 17, 66-72, 1980

8. Hummel, J.P. and W.J. Dreyer. Measurement of protein-binding phenomena by gel filtration. Biochem. Biophys. Acta 63, 530-532, 1962 
9. Maclean-Fletcher, S.D. and T.D. Pollard. Viscometric analysis of the gelation of Acanthamoeba extracts and purification of two gelation factors. J. Cell Biology 85, 414-428, 1980

10. Maeda, H., N. Ishida, H. Kawauchi and K. Tsuzimura. Reaction of fluorescein-isothiocyanate with proteins and amino acids. I. Covalent and non-covalent binding. J. Biochem. 65, 777-783, 1969

11. Maeda, H., M. Nakayama, D. Iwaoka and T. Sato. Assay of angiotensin I by fluorescence polarization method. in KININS-II: Biochemistry, Pathophysiology, and Clinical Aspects. ed. S. Fujii, H. Moriya and T. Suzuki, Plenum Pub. Corp. New York, pp. 203-211, 1979

12. Maeda, H. Assay of proteolytic enzymes by fluorescence polarization technique. Anal. Biochem. 92, 222-237, 1979

13. Maeda, H., H. Tsuda and J. TAKeshita. Investigation of factors involved in the uptake velocity of fluorescein diacetate and intracellular fluorescence polarization value. II. Cytotoxicity produced by anticancer agents. Cell Structure and Function 7, 177-182, 1982

14. Means, A.R. and J.R. Dedman. Calmodulin-An intracellular calcium receptor. Nature 285, 73-77, 1980

15. Pittinger, C. and R. Adamson. Antibiotics blockade of neuromuscular function. Ann. Rev. Pharmacol. 12, 169-184, 1972

16. Putney, Jr., J.W. Stimulus-permeability coupling: Role of calcium in the receptor regulation of membrane permeability. Pharmacol. Rev. 30, 209-245, 1979

17. Rotman, B. and B.W. Papermaster. Membrane properties of living mammalian cells as studied by enzymatic hydrolysis of fluorogenic esters. Proc. Natl. Acad. Sci. U.S.A. 55, 134 141,1966

18. Rotman, A. and J. Heldman. Intracellular viscosity changes during activation of blood platelets: Studies by fluorescence polarization. Biochemistry 20, 5995-5999, 1981

19. Sakamoto, S., H. Maeda, K. Yamasaki, S. Aikawa and Y. Hinuma. Evaluation of cellular fluidity of peripheral blood lymphocytes from patients with urogenital cancers by a fluorescence polarization technique: PHA response and prognosis. Eur. J. Cancer and Clin. Oncol. 17, 11051115,1981

20. SLOBODA, R.D. The role of microtubules in cell structure and cell division. American Scientist 68, 290-298, 1980

21. TakakU, F., T. Yamanaka and Y. Hashimoto. Usefulness of the SCM test in the diagnosis of gastric cancer. Br. J. Cancer 36, 810-813, 1977

22. Tamura, Y., M. Hirado, K. Okamura, Y. Minato and S. Fujil. Synthetic inhibitors of trypsin, plasmin, kallikrein, thrombin, $\mathrm{C}_{1} \mathrm{r}$, and $\mathrm{C}_{1}$ esterase. Biochem. Biophys. Acta 484, 417422, 1977

23. Tsuda, H., H. MAEDA and S. Kishimoto. Fluorescence polarization with FDA in leukemic cells: A clear difference between myelogenous and lymphocytic origins. Br. J. Cancer 43, 793-803, 1981

24. UdKoFf, R. and A.J. NoRman. Polarization of fluorescein fluorescence in single cells. Histochem. Cytochem. 27, 49-55, 1979

25. Umezawa, H. Structures and Activities of Protease Inhibitors of Microbial Origin. Methods in Enzymology XLV, 678-695, 1976 